\title{
Study to Evaluate the Conditions Leading to Diagnosis of Type 2 Diabetes Mellitus in Women in a Medical College in Kolkata, West Bengal, India
}

\author{
${ }^{1}$ Nirmalya Roy, ${ }^{2}$ Ankan Pathak, ${ }^{3}$ Esha Gupta, ${ }^{4}$ Kingshuk Bhattacharjee, ${ }^{5}$ Ashis Saha
}

\begin{abstract}
Diabetes is one of the most important public health problems. There is paucity of Indian data as to which conditions leads to diagnosis of type 2 diabetes mellitus (T2DM). The present study endeavors to throw some light on the problem. A cross-sectional and retrospective study including 321 subjects, performed at a tertiary care hospital in eastern India A total of 321 female patients diagnosed with diabetes over a period of 24 months were included. Mean age at diagnosis being $45.54 \pm 10.8$ years, respectively. Out of these, only $23.05 \%$ presented with osmotic and other symptoms (foot ulcer, weight loss, etc.) suggestive of diabetes. A substantial $43.52 \%$ of total patients were diagnosed during general healthcare check-up [35.2\% in outpatient department (OPD) and $8.32 \%$ during perioperative evaluation]. Remaining patients diagnosed with diabetes included $8.28 \%$ patients admitted with acute coronary syndrome, $13.4 \%$ with infections of different types and $11.75 \%$ with other non-related diseases. Among diagnosed diabetics, $50.47 \%$ had HTN and $41.12 \%$ had IHD.
\end{abstract}

Keywords: Diabetes, KPC Medical College and Hospital, Jadavpur, Women.

How to cite this article: Roy N, Pathak A, Gupta E, Bhattacharjee K, Saha A. Study to Evaluate the Conditions Leading to Diagnosis of Type 2 Diabetes Mellitus in Women in a Medical College in Kolkata, West Bengal, India. Bengal Physician Journal, 2018;5(3):29-31.

\section{Source of support: Nil \\ Conflict of interest: None}

\section{INTRODUCTION}

\section{So..Why Women???}

- Far-reaching changes in the hormonal milieu. ${ }^{1}$

- Juggling the roles of a homemaker and professional life.

- Complicates pregnancy.

\footnotetext{
${ }^{1}$ Professor, ${ }^{2}$ Postgraduate Trainee, ${ }^{3}$ Resident, ${ }^{4}$ Statistician, ${ }^{5}$ Professor and Head

1-3,5 Department of Medicine, KPC Medical College and Hospital, Kolkata, West Bengal, India

${ }^{4}$ Department of Medical Sciences, SJJT University, Jhunjhunu, Vidyanagari, Rajasthan, India
}

Corresponding Author: Ankan Pathak, Postgraduate Trainee, Department of Medicine, KPC Medical College and Hospital, Kolkata, West Bengal, India, e-mail: ankan.manu@gmail.com
- Menopause brings about severe anxiety and depression.

- Diabetes is associated with certain cancers in women.

- Certain drugs are best avoided in diabetic women.

\section{AIM}

- There is currently a paucity of data on women with diabetes.

- The main aim of this study was to evaluate the various conditions which lead to the diagnosis of diabetes in women, the age group in which the diagnosis is most common and the associated comorbidities, if any.

\section{METHODS}

A cross-sectional study utilized administrative data of KPC Medical College and Hospital, Jadavpur and BM Birla Hospital accumulated over 6 months.

Diabetes is diagnosed according to the American Diabetes Association (ADA) diagnostic criteria with the presence of any one or more of the following:

- Fasting plasma glucose concentration $\geq 126 \mathrm{mg} / \mathrm{dL}$.

- Plasma glucose concentration $>200$ mg/dL.

- Symptoms of hyperglycemia.

- Random plasma glucose concentration $>200 \mathrm{mg} / \mathrm{dL}$.

- Glycosylated hemoglobin $>6.5 \%$.

- Study period: 6 months.

- Sample size: 321 patients (indoor and outdoor).

- Written informed consent was taken from patients.

\section{Inclusion Criteria}

- Individuals reporting that they were not diagnosed with diabetes earlier.

- Individuals who consented to share their data.

\section{Analysis}

- Descriptive statistical analysis was carried out.

- Significance was assessed at the 5\% level.

- Statistical analysis system (SAS) version 9.2 for windows and statistical package for the social sciences (SPSS) version 20.0 was used for the analysis of the data.

- Microsoft Word and Excel was used to generate graphs and tables. 


\section{RESULTS}

- The study sample consisted of a total of 321 patients diagnosed with T2DM over 6 months.

- Mean age at diagnosis was 45.54 plus minus 10.8 years.

- The mean age of the study population was 54.7 years.

- Maximum number of subjects (31.78\%) belonged to the sixth decade age group followed by $23.68 \%$ and $24.61 \%$ of the seventh and the fourth decade, respectively (Graph 1).

\section{Health Conditions Leading to Diagnosis}

\section{Baseline Characteristics}

- Age

- BMI (Graph 2)

- Religion (Graph 3)

- Association between age with HTN and IHD in women diabetic subjects

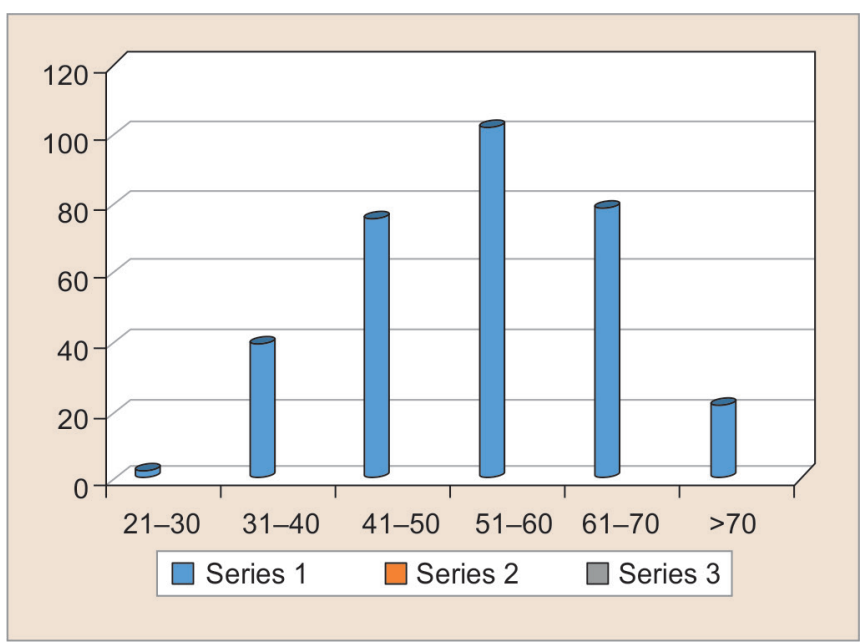

Graph 1: Diagnosis of diabetes of various age groups

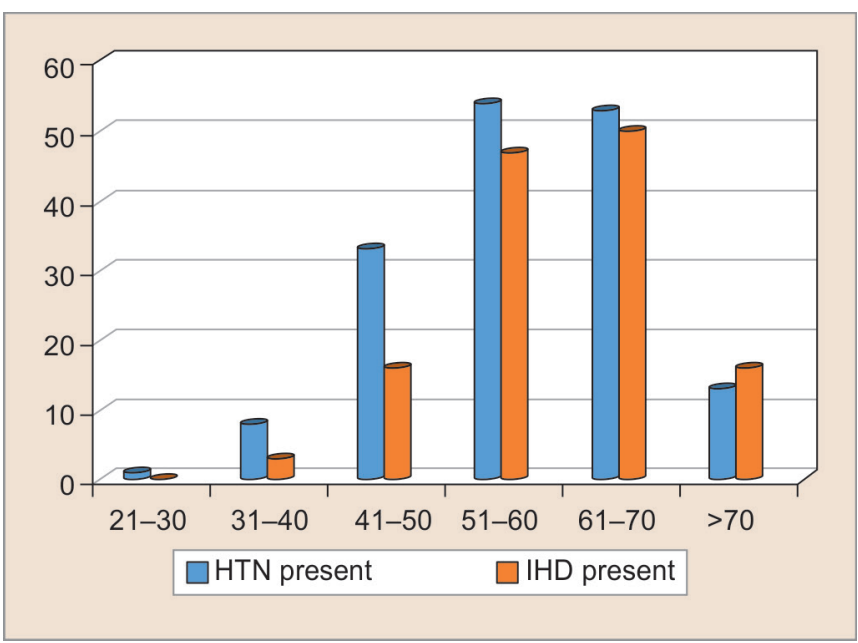

Graph 3: Diagnosis of type 2 diabetes mellitus in women of different religion

\section{DISCUSSION}

- Type 2 diabetes mellitus can be present for 9-12 years before being diagnosed and in most of cases, complications are often present at the time of diagnosis.

- The prime reason for the late detection happens to be the absence of classical osmotic symptoms.

- In our study, we found only $23.05 \%$ of subjects presented with osmotic symptoms (Graph 4). Thus the remaining $77 \%$ subjects were asymptomatic for diabetes.

- The UKPDS VIII had demonstrated that $50 \%$ of patients had diabetes-related tissue damage at the time of diagnosis. $^{2}$

- This observation is in the same line with our findings, as IHD were present in $42.12 \%$ of our subjects (Graph 5). Around $8.28 \%$ of study subjects were diagnosed with T2D when admitted with acute coronary syndrome.

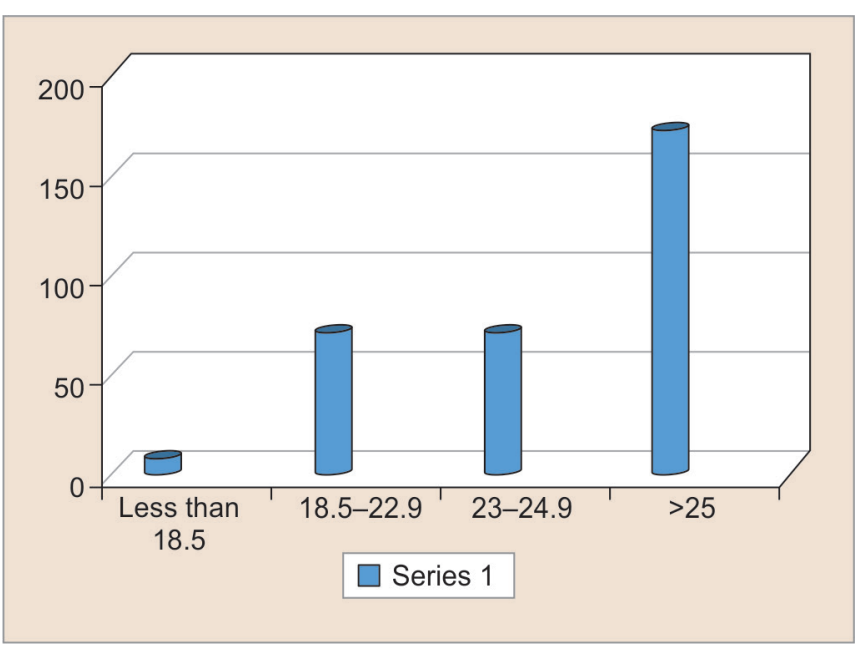

Graph 2: Association of type 2 diabetes mellitus with various $\mathrm{BMI}$

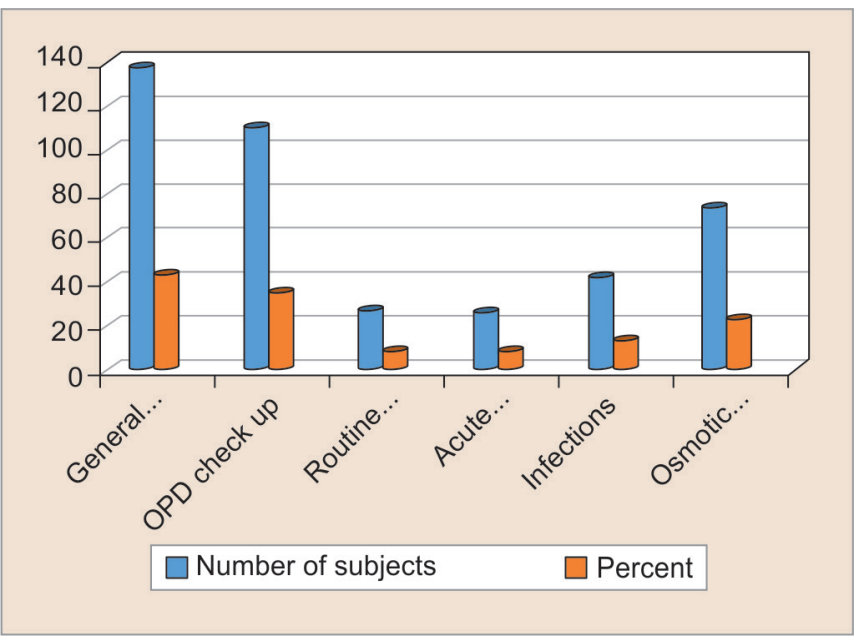

Graph 4: Condition leading to diagnosis of diabetes in women 


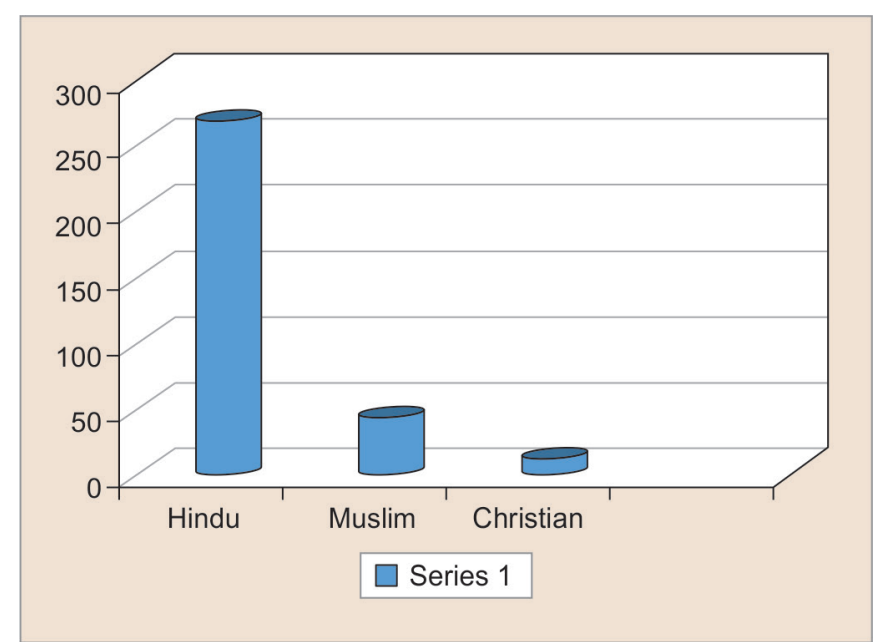

Graph 5: Association of hypertension and IHD at the time of diagnosis of type 2 diabetes mellitus
- As the health of the women is central to the health of the family and therefore the nation diabetes in women deserves more attention than it currently receives. $^{3}$

- Diabetes is as common in women as in men, but the former tend to encounter greater difficulties controlling their blood glucose levels. ${ }^{4}$

\section{REFERENCES}

1. www.cdc.gov.

2. https://www.ncbi.nlm.nih.gov.

3. RSSDI update 2015, chapter 15 and 16.

4. International Diabetes Federation. Diabetes and women Available from www.odf.org/diabetes and women. 